\title{
Avaliação do Impacto Ambiental Utilizando Ferramentas Físico-Químicas
}

Environmental Impact Assessment Using Physical-Chemical Tools

Evaluación del Impacto Ambiental Utilizando Herramientas Físico-Químicas

Gilmar de Almeida Gomes

Carolina Franchini

Eliziane Zambiasi 


\section{RESUMO}

Em vista dos problemas atuais relacionados a água própria para consumo humano, é necessário avaliar e preservar os recursos hídricos disponíveis. Este artigo apresenta os resultados de análises físico-químicas (cloreto, dureza, alcalinidade e pH) para os rios Lajeado Bonito e Limeira da cidade de Pinhalzinho, Oeste de Santa Catarina e também os cálculos de Energia de Gibbs para cada ponto avaliado. Também foram comparados os resultados obtidos com os anteriores de 2015. Notou-se uma piora em relação a maioria dos pontos para os dois rios, indicando uma maior ação antropogênica sobre eles. Houve aumento principalmente no teor de cloreto dos dois rios, indicando maior carga de esgotos domésticos lançados nestes pontos. Ocorreu variação na Energia de Gibbs para todos os pontos. Quanto menor a Energia de Gibbs, mais estável é o sistema, mas essa estabilidade do sistema vem à custa de uma vizinhança com maior energia para evitar o desequilíbrio ambiental.

PALAVRAS-CHAVE: Rio Lajeado Bonito. Rio Limeira.Íons.

\section{SUMMARY}

In view of the current problems related to water for human consumption, it is necessary evaluate and preserve the available water resources. This study presents the results of physico-chemical analyzes (chloride, hardness, alkalinity and $\mathrm{pH}$ ) for the rivers Lajeado Bonito and Limeira of the city of Pinhalzinho, West of Santa Catarina and also Gibbs Energy calculations for each evaluated point. The results obtained with the previous ones of 2015 were also compared. It was noticed a worsening in relation to most points for the two rivers, indicating a greater anthropogenic action on them. There was an increase mainly in the chloride content of the two rivers, indicating a higher load of domestic sewage released at these points. Gibbs Energy varied for all points. The lower the Gibbs Energy, the more stable the system is, but this stability of the system comes at the expense of a neighborhood with greater energy to avoid environmental imbalance.

KEYWORDS: River Lajeado Bonito. River Limeira. lons.

\section{RESUMEN}

En vista de los problemas actuales relacionados con el agua propia para el consumo humano, es necesario evaluar y preservar los recursos hídricos disponibles. Este artículo presenta los resultados de análisis físico-químicos (cloruro, dureza, alcalinidad y $\mathrm{pH}$ ) para los ríos Lajeado Bonito y Limeira de la ciudad de Pinhalzinho, Oeste de Santa Catarina y también los cálculos de Energía de Gibbs para cada punto evaluado. También se compararon los resultados obtenidos con los anteriores de 2015. Se notó un empeoramiento en relación a la mayoría de los puntos para los dos ríos, indicando una mayor acción antropogénica sobre ellos. Se observó un aumento principalmente en el contenido de cloruro de los dos ríos, indicando mayor carga de alcantarillas domésticas lanzadas en estos puntos. Se produjo variación en la Energía de Gibbs para todos los puntos. Cuanto menor es la Energía de Gibbs, más estable es el sistema, pero esta estabilidad del sistema viene a costa de una vecindad con mayor energía para evitar el desequilibrio ambiental.

PALABRAS CLAVE: Río Lajeado Bonito. Río Limeira. lones. 


\section{INTRODUÇÃO}

Água é um recurso essencial para qualquer atividade humana, pois além de ser fundamental para a sobrevivência de qualquer organismo vivo, segundo Farias (2006) ela também é padrão de qualidade de vida de uma população, sendo o recurso mais crítico a impor limites ao desenvolvimento de uma região.

Com o passar do tempo, a crescente densidade demográfica aliada ao aumento da produção tanto industrial, como na agricultura, sem um planejamento prévio de seus resíduos vem gerando grandes estragos na qualidade das águas, pois a mesma não consegue acompanhar esse ciclo que se torna cada vez mais rápido, causando assim, desequilíbrio em todo o ecossistema.

A região Oeste de Santa Catarina caracteriza-se pelas cadeias de produção agropecuária: leite, carne suína, aves e em menor quantidade carne bovina, juntamente com a produção de grãos. Essas atividades iniciam nas propriedades rurais, gerando todo um resíduo orgânico característico juntamente com produtos químicos e defensivos agrícolas.

Sendo que na maioria das situações todos esses resíduos não são tratados de modo satisfatório e acabam contaminando todo o sistema hídrico. Podendo se verificar através dos parâmetros físicos, químicos e biológicos a relação dos efeitos antrópicos, através da qualidade da água e suas interações.

O objetivo desse trabalho é avaliar, através de parâmetros físico-químicas e cálculos de Variação de Energia de Gibbs, como o sistema hídrico responde a essas atividades através de análises realizadas em dois rios da cidade de Pinhalzinho- SC, sendo o rio Limeira e Lajeado Bonito em diferentes pontos de coleta na área urbana e rural.

\section{MATERIAIS E MÉTODOS}

\subsection{Análises}

As coletas foram realizadas em pontos específicos de cada rio no período de julho de 2016 a dezembro de 2016. Realizadas em frascos âmbar previamente esterilizados e as amostras mantidas sob refrigeração até o momento da análise. As análises foram realizadas no laboratório de Química Geral e Química de Alimentos do Centro de Educação Superior do Oeste - UDESC/ Pinhalzinho, SC.

A temperatura foi medida com termômetro no momento da coleta e o restante das análises foram realizadas no laboratório, sendo elas:

- $\mathrm{pH}$ : realizada em pHmetro de bancada Q400 MT da marca Quimis. O pH é usado para expressar a intensidade da condição ácida ou básica de uma solução e é uma maneira de expressar a concentração do íon hidrogênio, sendo muito influenciado pela quantidade de matéria em decomposição, quanto maior a quantidade de matéria 
orgânica disponível, menor o $\mathrm{pH}$, pois para haver decomposição de materiais muito ácido são produzidos (FARIAS, 2006).

- Cloreto por titulação argentométrica. A concentração de cloreto nas águas pode ser resultado do contato da água com depósitos minerais, com a água do mar, poluição por esgotos (domésticos e industriais) ou ao retorno de águas utilizadas em irrigação agrícola.

- A dureza foi analisada por titulação de complexação, utilizando Ériocromo T como indicador. Indicando a concentração de cálcio e magnésio;

- A Alcalinidade foi medida por titulação de neutralização usando fenolftaleína como indicador. A alcalinidade da água é uma medida de sua capacidade de neutralizar ácido e é devida, principalmente a sais de ácidos fracos e bases fortes, e tais substâncias têm efeito tampão, resistindo à queda de $\mathrm{pH}$ resultante da adição de ácidos. A porção principal de alcalinidade em águas naturais é causada por hidróxidos, carbonatos e bicarbonatos. A determinação e controle da alcalinidade tomam-se importantes na avaliação da qualidade das águas com o objetivo de evitar problemas de corrosão e de incrustação nas tubulações (FARIAS, 2006).

\subsection{Equações}

Utilizando para calcular a Energia de Gibbs de cada os íons especifico sendo eles: cloreto, dureza (cálcio e magnésio) e alcalinidade (bicarbonato) e da relação total desses íons em cada ponto, a equação citadas por Atkins e De Paula (2012).

$$
\Delta m G=n \cdot R \cdot T(x a \cdot \ln x a+x b \cdot \ln x b)
$$

- $\mathrm{n}$ = número de mols;

- $\mathrm{R}=8,314 \mathrm{~J} / \mathrm{mol} . \mathrm{K}$

- $\mathrm{T}=$ temperatura em Kelvin;

- $\quad x$ a ou $x \mathrm{~b}=$ fração molar.

A separação entre cálcio e magnésio para a dureza foi realizada utilizando os cálculos citados por Abdalla et al. (2010), sendo:

$$
\begin{gathered}
\frac{m g}{L} C a=\frac{m l \text { de EDTA } \times 400,8}{\text { volume de amostra }} \\
\frac{m g}{L} M g=\frac{\text { dureza }\left(\frac{m g}{L}\right)-2,497}{\text { volume da amostra }}
\end{gathered}
$$




\section{Periódica Eletranica}

\section{Fórum Ambiental}

Volume 14, Número 1, 2018

da Alta Paulista

ISSN 1980-0827

\section{3 Área de coleta}

Com o intuito de avaliar as diferentes relações entre a ação antropogênica, coletou-se água de diferentes pontos, localizados em áreas urbanas e rurais rios Lajeado Bonito e Limeira.

Foram realizadas 5 coletas para cada rio. No rio Lajeado Bonito, foram definidos 4 pontos de coleta, sendo 2 urbanos e 2 rurais.

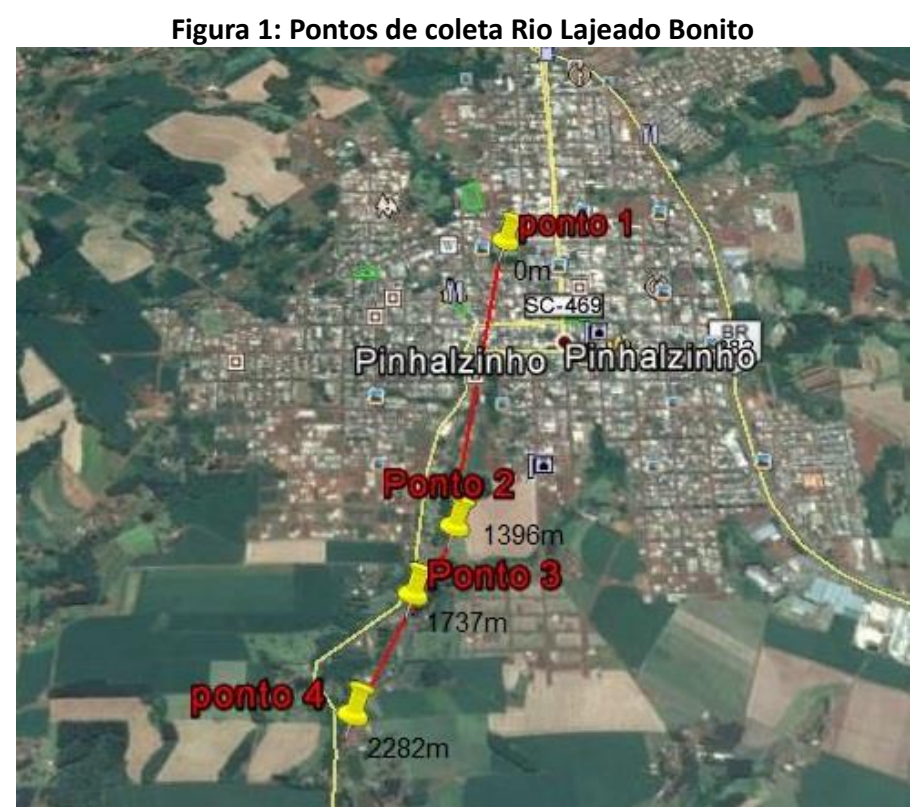

Fonte: Google Earth, 2017.

No rio Limeira foram definidos também 4 pontos de coleta sendo 1 urbano e 3 rurais.

Figura 2: Pontos de coleta Rio Limeira

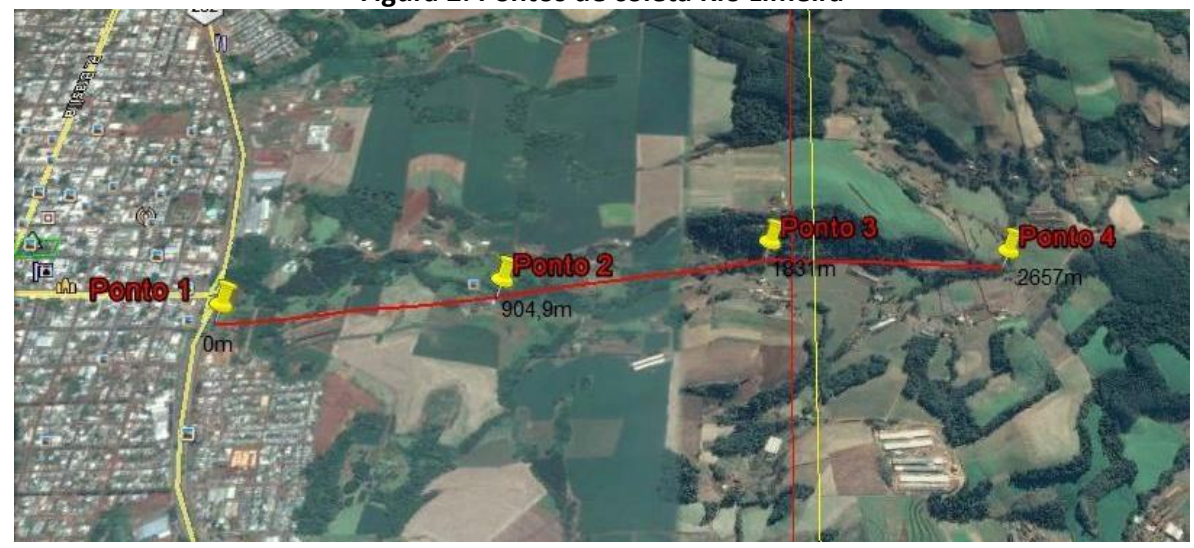

Fonte: Google Earth, 2017.

\section{RESULTADOS E DISCUSSÕES}




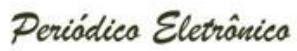 \\ Fórum Ambiental
}

Volume 14, Número 1, 2018

ISSN 1980-0827

\author{
da Alta Paulista
}

\section{Rio Lajeado Bonito}

Na tabela 1 apresenta-se o resultado das análises físico-químicas para os quatro pontos do Rio Lajeado Bonito, comparando os resultados de 2015 e 2016.

Fonte: Dados da pesquisa, 2016.

Os pontos 01 e 02 estão localizados mais próximos a área urbana, recebendo uma maior carga de contaminantes antropogênicos, como esgotos domésticos e industriais. Nota-se que houve redução nos valores de cloreto para o ponto 02 e 03, o que indica uma dissolução dos íons na água. Nota-se também uma redução na dureza, o que indica redução dos íons cálcio e magnésio. O ponto quatro apresentou elevação no teor de cloreto e alcalinidade, este ponto está localizado no perímetro rural. Isso indica uma elevação de contaminantes na água, isto pode ter ocorrido devido ao aumento do uso de fertilizantes, levando a maior contaminação.

A Tabela 2 apresenta os cálculos de Energia livre de Gibbs para cada íon analisado e a e para a mistura desses íons em cada ponto.

\begin{tabular}{|c|c|c|c|c|c|c|c|c|c|c|}
\hline \multicolumn{11}{|c|}{ Tabela 01 - Análises Físico químicas } \\
\hline & \multicolumn{2}{|c|}{$\begin{array}{c}\text { Temperatura } \\
\left({ }^{\circ} \mathrm{C}\right)\end{array}$} & \multicolumn{2}{|c|}{ Cloreto (mg/L) } & \multicolumn{2}{|c|}{ Dureza (mg/L) } & \multicolumn{2}{|c|}{$\begin{array}{c}\text { Alcalinidade } \\
(\mathrm{mg} / \mathrm{L})\end{array}$} & \multicolumn{2}{|c|}{ pH } \\
\hline & 2015 & 2016 & 2015 & 2016 & 2015 & 2016 & 2015 & 2016 & 2015 & 2016 \\
\hline Ponto 01 & 22,3 & 17,9 & 5,02 & 8,15 & 160,1 & 46,9 & 33,0 & 33,25 & 7,25 & 7,60 \\
\hline Ponto 02 & 21,0 & 19 & 10,53 & 7,61 & 88,1 & 37,8 & 32,0 & 22,53 & 7,18 & 6,88 \\
\hline Ponto 03 & 20,8 & 17,3 & 7,72 & 7,06 & 185,4 & 33,5 & 33,0 & 24,88 & 7,32 & 7,01 \\
\hline Ponto 04 & 20,5 & 19,6 & 4,93 & 6,90 & 160,1 & 40,5 & 22,0 & 39,75 & 7,25 & 7,53 \\
\hline
\end{tabular}

Tabela 02 - Cálculo da Energia Livre de Gibbs

\begin{tabular}{|c|c|c|c|c|c|c|c|c|c|c|}
\hline & \multicolumn{2}{|c|}{$\Delta \mathrm{mG}\left(\mathrm{Cl}^{-}\right)(\mathrm{J})$} & \multicolumn{2}{|c|}{$\begin{array}{c}\Delta \mathrm{mG}\left(\mathrm{Ca}^{++}\right) \\
\left(\mathrm{J} 10^{-3}\right)\end{array}$} & \multicolumn{2}{|c|}{$\begin{array}{c}\Delta \mathrm{mG}\left(\mathrm{Mg}^{++}\right) \quad(\mathrm{J} \\
\left.10^{-3}\right)\end{array}$} & \multicolumn{2}{|c|}{$\begin{array}{c}\Delta \mathrm{mG}\left(\mathrm{HCO}^{3-}\right) \\
\left(\mathrm{J} 10^{-2}\right)\end{array}$} & \multicolumn{2}{|c|}{$\begin{array}{c}\Delta \mathrm{mG} \text { (total) } \\
\left(\mathrm{J} \mathrm{10^{-2 } )}\right.\end{array}$} \\
\hline & 2015 & 2016 & 2015 & 2016 & 2015 & 2016 & 2015 & 2016 & 2015 & 2016 \\
\hline Ponto 01 & $-9,89 \times 10^{-3}$ & $-4,32 \times 10^{-3}$ & $-0,0362$ & $-8,151$ & $-5,30$ & $-1,59$ & $-0,0221$ & $-2,35$ & $-8,45 \times 10^{-3}$ & $-1,56$ \\
\hline Ponto 02 & $-0,0264$ & $-0,01827$ & $-6,99 \times 10^{-3}$ & $-7,593$ & $-2,19$ & $-1,21$ & $-0,0214$ & $-1,42$ & $-0,0363$ & $-0,712$ \\
\hline Ponto 03 & $-0,0180$ & $-0,0165$ & $-0,0195$ & $-6,789$ & $-6,38$ & $-1,02$ & $-0,0220$ & $-1,61$ & $-0,0151$ & $-1,25$ \\
\hline Ponto 04 & $-9,66 \times 10^{-3}$ & $-0,1601$ & $-0,0113$ & $-7,945$ & $-5,27$ & $-1,33$ & $-0,0125$ & $-2,90$ & $-0,0357$ & $-1,27$ \\
\hline
\end{tabular}

Fonte: Dados da pesquisa, 2016.

O ponto dois continuou apresentando a menor Energia de Gibbs total, sendo o mais estável em comparação aos pontos analisados, porém está situado na área atribuída como mais contaminada, recebendo forte ação antropogênica. Segundo as leis da termodinâmica a tendência do sistema e vizinhança é sempre estar em equilíbrio, assim, se o sistema (rio) possui uma menor energia, a vizinhança deve possuir uma maior energia para manter o sistema em 


\section{Periódica Eletrânica}

\section{Fórum Ambiental}

equilíbrio. Estabilidade termodinâmica do sistema, somente, não indica equilíbrio do universo (sistema mais vizinhança).

Nota-se que a Energia Livre de Gibbs para o cálcio aumentou para a maioria dos pontos, porém diminui para todos eles em termos de magnésio. Isso auxilia a entender a redução na dureza apresentada anteriormente.

Além disso, como visto na tabela 01 , a alcalinidade associada ao íon $\mathrm{HCO}^{3-}$ aumentou nas análises físico químicas e também na Energia Livre de Gibbs.

\section{Rio Limeira}

Na tabela 3 são apresentados os resultados das análises físico-químicas para os pontos de coleta do Rio Limeira em comparação com o ano de 2015.

Fonte: Dados da pesquisa, 2016.

Para esse rio fica bem mais nítido de se observar que há uma maior influência da ação antropogênica no ponto um que seria o ponto urbano, pois além da concentração dos íons de cloreto e alcalinidade ser maior o $\mathrm{pH}$ também é o menor.

O ponto um demonstrou piora em relação ao ano anterior pois houve aumento nos valores de cloreto, alcalinidade e redução de pH. Este ponto está recebendo uma carga ainda maior de contaminantes levando a desequilíbrio do sistema.

Além disso, houve aumento no teor de cloreto em todos os pontos, indicando que este rio está recebendo uma maior carga de contaminantes, possivelmente esgotos domésticos.

Na tabela 4 são apresentados os cálculos para Energia Livre de Gibbs para cada íon e a para a mistura desses íons para todos os pontos do Rio Limeira.

\begin{tabular}{|c|c|c|c|c|c|c|c|c|c|c|c|c|c|}
\hline \multicolumn{14}{|c|}{ Tabela 03 - Análises Físico químicas } \\
\hline & & \multicolumn{3}{|c|}{ Temperatura $\left({ }^{\circ} \mathrm{C}\right)$} & \multicolumn{2}{|c|}{ Cloreto $(\mathrm{mg} / \mathrm{L})$} & \multicolumn{2}{|c|}{ Dureza (mg/L) } & \multicolumn{2}{|c|}{$\begin{array}{c}\text { Alcalinidade } \\
(\mathrm{mg} / \mathrm{L})\end{array}$} & \multicolumn{3}{|c|}{$\mathrm{pH}$} \\
\hline & & \multicolumn{2}{|c|}{2015} & 2016 & 2015 & 2016 & 2015 & 2016 & 2015 & 2016 & 2015 & \multicolumn{2}{|c|}{2016} \\
\hline \multicolumn{2}{|c|}{ Ponto 01} & \multicolumn{2}{|c|}{19,5} & 19,7 & 7,62 & 9,94 & 93,3 & 32,5 & 25,0 & 32,3 & 7,13 & \multicolumn{2}{|c|}{6,94} \\
\hline \multicolumn{2}{|c|}{ Ponto 02} & \multicolumn{2}{|c|}{20,4} & 18,1 & 8,13 & 8,61 & 91,3 & 40,5 & 28,0 & 25,8 & 6,85 & \multicolumn{2}{|c|}{7,47} \\
\hline \multicolumn{2}{|c|}{ Ponto 03} & \multicolumn{2}{|c|}{19,0} & 17,6 & 2,92 & 6,68 & 144,1 & 27,5 & 26,0 & 24,3 & 6,96 & & \\
\hline Pontc & & &, 5 & 16,6 & 4,94 & 6,30 & 163,4 & 27,5 & 24,0 & 22,8 & 7,01 & & \\
\hline & & & & & Tabela 04 & culo c & Energia & ivre de $C$ & ibbs & & & & \\
\hline & & $\mathrm{mG}\left(\mathrm{Cl}^{-}\right.$ & & & $\begin{array}{r}\Delta \mathrm{mG}(\mathrm{C} \\
\left(\mathrm{J} 10^{-}\right.\end{array}$ & & $\begin{array}{r}\Delta m G(n \\
\text { (J 10 }\end{array}$ & & $\begin{array}{r}\Delta m G(H C \\
\text { (J 10- }\end{array}$ & & $\begin{array}{r}\Delta \mathrm{mc} \\
\text { (J }\end{array}$ & 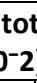 & \\
\hline & & 2015 & 2016 & & 2015 & 2016 & 2015 & 2016 & 2015 & 2016 & & & 2016 \\
\hline Ponto 01 & & 0,0191 & $-2,49$ & & $-0,0179$ & $-5,85$ & $-2,86$ & $-1,04$ & $-0,0171$ & $-2,33$ & $-9,77 x$ & & 1,12 \\
\hline Ponto 02 & & 0,0205 & $-2,08$ & & $-0,0176$ & $-15,0$ & $-2,75$ & $-1,39$ & $-0,0198$ & $-1,77$ & $-3,56 x$ & & 4,51 \\
\hline Ponto 03 & -5 & $9 \times 10^{-3}$ & $-1,49$ & & $-0,0315$ & $-5,89$ & $-5,07$ & $-0,82$ & $-0,0180$ & $-1,63$ & $-8,46 x$ & & $-1,68$ \\
\hline
\end{tabular}




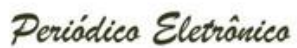

\begin{tabular}{l|l|l|l|l|l|l|l|l|l|l} 
Ponto 04 & $-0,0111$ & $-1,38$ & $-0,0364$ & $-5,86$ & $-5,89$ & $-0,81$ & $-0,1062$ & $-1,49$ & $-0,0207$ & $-1,77$ \\
\hline
\end{tabular}

Fonte: Dados da pesquisa, 2016.

Nota-se que o ponto quatro apresenta a menor Energia de Gibbs total de mistura, sendo assim o mais estável. Este ponto está situado na área rural, indicando menor contaminação antropogênica.

Como dito na tabela anterior, houve aumento em todos os pontos em teores de cloreto. Podese perceber também que a estabilidade neste rio está afetada pois houve aumento na Energia de Gibbs de mistura em todos os parâmetros, indicando um desequilíbrio no sistema.

\section{CONCLUSÃO}

Notou-se uma piora nos parâmetros avaliados, indicando maior impacto ambiental sobre os rios avaliados. Isso pode ter ocorrido devido ao aumento do lançamento de esgotos domésticos e industrias de maneira irregular nos rios. Não apenas os pontos urbanos foram atingidos, mas também os rurais, indicando que a dissolução dos íons levou a aumento de concentração nos pontos mais afastados do centro urbano.

\section{REFERÊNCIAS BIBLIOGRÁFICAS}

ABDALLA, K. V. P. et al. Avaliação da Dureza e das Concentrações de Cálcio e Magnésio em Água Subterrâneas da Zona Urbana e Rural do Município de Rosário - MA. XVI Congresso Brasileiro de Águas Subterrâneas, São Luís, 2010. Disponível em:< file:///C:/Users/User/Downloads/22915-82903-1-PB.pdf>. Acesso em: 10 mai. 2017.

ATKINS, P. W.; PAULA, J. de. Físico-Química. Ed.9., v.1, 2010.

BRASIL. Ministério da Saúde. Dispõe sobre os procedimentos de controle e de vigilância da qualidade da água para consumo humano e seu padrão de potabilidade. Portaria n. 2914, de 12 de dezembro de 2011. Brasília, 2011. Disponível em:<http://bvsms.saude.gov.br/bvs/saudelegis/gm/2011/prt2914_12_12_2011.html>. Acesso em: 04 abr. 2017.

CONSELHO NACIONAL DO MEIO AMBIENTE - CONAMA. Resolução no 357 de 17 de março de 2005. Dispõe sobre a classificação dos corpos de água e diretrizes ambientais para o seu enquadramento, bem como estabelece as condições e padrões de lançamento de efluentes. Ministério do Meio Ambiente. 2005.

FUNASA. Fundação Nacional de saúde. Manual Prático de Análise de Água. Acessória de comunicação e educação em saúde. Brasília/DF. Disponível em:<www.funasa.gov.br>. Acesso em 11 mai. 2017.

GOOGLE. Google Earth. Version X. 2016. Pinhalzinho - SC. Disponível em: < https://www.google.com/earth/>. Acesso em: 10 jun. 2017.

$\mathrm{KIM}$, K. L. et al. Inorganic chemicals in an effluent-dominated stream as indicators for chemical reactions and streamflows. Journal of Hidrology. 2002.

MARQUEZ, M. N. et al. Avaliação do impacto da agricultura em áreas de proteção ambiental, pertencentes à bacia hidrográfica do Rio Ribeira de Iguape, São Paulo. Química Nova, São Paulo, v.30, n.5, p. 1171-1178, 2007. Disponível em:< http://www.scielo.br/scielo.php?script=sci_arttext\&pid=S0100-40422007000500023>. Acesso em: 03 jun. 2017.

MAYER, D. A. et al. Medidas da Variação da Energia de Gibbs como efeito antropogênico. Um estudo comparativo entre Área Urbana e Rural. IX Congresso Brasileiro de Engenharia Química. Maringá - PR. 2012. 